\title{
UTILIZAÇÃO DE PACIENTES SIMULADOS NO ENSINO E NA AVALIAÇÃO DE HABILIDADES CLÍNICAS
}

\author{
UTILIZATION OF SIMULATED PATIENTS FOR CLINICAL SKILLS TEACHING AND ASSESSMENT
}

\author{
Luiz Ernesto de Almeida Troncon
}

Docente. Departamento de Clínica Médica. Faculdade de Medicina de Ribeirão Preto - USP.

CorRespondÊncia: Departamento de Clínica Médica - Hospital das Clinicas da Faculdade de Medicina de Ribeirão Preto - Campus da USP - 14 048-900. Ribeirão Preto - SP. Telefone: (16) 36022457 Fax: (16 3633 6695) E-mail: ledatron@fmrp.usp.br

Troncon LEA. Utilização de pacientes simulados no ensino e na avaliação de habilidades clínicas. Medicina (Ribeirão Preto) 2007;40 (2): 180-91.

Resumo: A utilização de pessoas normais desempenhando o papel de pacientes vem sendo feita há várias décadas no ensino e na avaliação de habilidades clínicas, como um meio de contornar os inconvenientes da utilização dos pacientes reais. No âmbito do ensino, o paciente simulado constitui um recurso complementar que pode anteceder a prática mais intensiva com os pacientes reais, permitindo que as atividades educacionais sejam desenvolvidas repetidamente com mais segurança e menor desgaste para os estudantes e os pacientes. Favorece, ainda, o aprendizado mais ativo, confere maior homogeneidade nas oportunidades de treinamento, permite repetições e facilita as correções dos defeitos na execução das tarefas com o paciente. No âmbito das avaliações, os pacientes simulados (padronizados) permitem a inserção nos exames das situações desejadas, no nível de complexidade apropriado, e que um grande número de examinandos seja avaliado nas mesmas condições, o que favorece o preenchimento dos requisitos de validade e de fidedignidade. Neste artigo, são apresentados conceitos educacionais pertinentes ao aprendizado das habilidades clínicas, um histórico sucinto do desenvolvimento das simulações empregando pessoas normais e as principais vantagens e desvantagens da utilização de pacientes reais e de pacientes simulados no ensino e na avaliação das habilidades clínicas. Alguns aspectos práticos do recrutamento, da seleção e do treinamento de pacientes simulados, baseados na experiência adquirida na Faculdade de Medicina de Ribeirão Preto, Universidade de São Paulo, são também apresentados.

Descritores: Simulação. Simulação de Pacientes. Pacientes Padronizados. Competência Clínica. Educação Médica.

\section{1- INTRODUÇÃO}

Nas últimas décadas, o ensino superior nas profissões da área da Saúde vem sofrendo inúmeras modificações conceituais e metodológicas visando o seu aperfeiçoamento. Uma destas modificações consiste na utilização crescente de pacientes simulados no ensino e na avaliação das habilidades clínicas. Idealizadas e desenvolvidas em centros da América do Norte, as técnicas de simulação tem se difundido por todo o mundo e vem sendo introduzidas e gradualmente empregadas com maior intensidade também no Brasil. Neste artigo, pretende-se apresentar as principais bases conceituais e discutir aspectos variados da utilização de pacientes simulados no ensino e na avaliação de habilidades clínicas, com o objetivo de divulgar estes conhecimentos para profissionais envolvidos no ensino médico e na educação superior na área da Saúde. 


\section{2- CONCEITO}

A formação do profissional da área da saúde e, em especial, a do médico, demanda sólido domínio das habilidades clínicas fundamentais, como a comunicação com pacientes, o exame físico, o raciocínio clínico e a proposição de medidas diagnósticas e terapêuticas. A atuação deste profissional poderá também exigir o adequado domínio de habilidades mais avançadas, entre as quais se incluem a execução propriamente dita de procedimentos diagnósticos ou terapêuticos de complexidade variável. A aquisição de proficiência nas habilidades clínicas depende do aprendizado adequado e, sobretudo, da prática reiterada. No caso de algumas habilidades, como as de comunicação, realização do exame físico e de procedimentos diagnósticos ou terapêuticos, a proficiência depende do exercício de tarefas que envolvem a participação de pessoas, sejam elas pacientes ou voluntários saudáveis, ou então objetos e dispositivos que simulam a situação clínica desejada para o tipo de habilidade a ser aprendida ou treinada.

No entanto, apesar da importância que o domínio das habilidades tem na definição da competência profissional, em muitas escolas, sobretudo nas mais tradicionais, o seu ensino, é pouco sistematizado ${ }^{1}$, distante das etapas e estratégias idealmente recomendadas (Quadro 1) e, portanto, com alta probabilidade de resultar em aprendizado deficiente ou heterogêneo.
O aprendizado das habilidades clínicas fundamentais, como a tomada da história clínica e a realização do exame físico, é tradicionalmente feito com o auxílio de pacientes reais, cuja saúde é assistida nas instituições que se responsabilizam pelo ensino. A participação dos pacientes reais no ensino e na avaliação, independentemente das considerações éticas que poderiam ser feitas, apresenta um número de vantagens e desvantagens (Quadro 2) que devem ser levadas em conta ${ }^{2}$. Em essência, os pacientes reais são muito adequados para o aprendizado das habilidades clínicas, pois apresentam problemas e situações reais e concretos e constituem recurso praticamente insubstituível para o ensino do exame físico. O custo da sua utilização educacional é muito baixo, pois já se encontra embutido no custo geral da assistência à sua saúde. No entanto, a utilização dos pacientes reais em atividades educacionais, ao lado dos inconvenientes do desgaste e do desconforto, apresenta inúmeras desvantagens do ponto de vista genuinamente educacional (Quadro 2), que devem ser considerados no planejamento do ensino.

O emprego de pessoas normais desempenhando o papel de pacientes constitui recurso desenvolvido há mais de quatro décadas ${ }^{3}$, que se firmou como uma possibilidade concreta de prover ensino e treinamento no campo das habilidades clínicas em função, fundamentalmente, do seu potencial de preencher condições mais próximas às ideais, bem como o de con-

Quadro 1: Principais etapas e estratégias que deveriam ser idealmente desenvolvidas para o sólido aprendizado das habilidades clínicas

\begin{tabular}{ll}
\hline ETAPAS & ESTRATÉGIAS \\
\hline 1. Conhecimento do que deve ser feito & Leituras, aulas \\
2. Verificação de como é feito & Demonstrações \\
3. Execução inicial da tarefa & Prática inicial com paciente \\
4. Verificação de erros na prática & Comentários de observador/avaliador \\
5. Preparação para nova tentativa & Reflexão \\
6. Re-execução da tarefa & Prática com paciente \\
7. Avaliação formativa do desempenho & Comentários de observador/avaliador \\
8. Treinamento & Exercício repetido da tarefa \\
9. Auto-avaliação & Reflexão \\
10. Avaliação somativa do desempenho & Exame prático formal de habilidades clínicas \\
\hline
\end{tabular}


Quadro 2. Vantagens e desvantagens educacionais da utilização de pacientes reais no ensino e na avaliação das habilidades clínicas, independentemente de considerações de ordem ética

\begin{tabular}{|c|c|c|}
\hline ATRIBUTOS & VANTAGENS & DESVANTAGENS \\
\hline $\begin{array}{l}\text { Adequação para } \\
\text { comunicação }\end{array}$ & $\begin{array}{l}\text { Alta, muitos pacientes comunicam- } \\
\text { se muito bem }\end{array}$ & $\begin{array}{l}\text { Pode ser baixa, uma vez que alguns paci- } \\
\text { entes têm dificuldades de comunicação }\end{array}$ \\
\hline $\begin{array}{l}\text { Adequação para } \\
\text { exame físico }\end{array}$ & Alta, pois apresentam sinais reais & $\begin{array}{l}\text { Ocasionalmente baixa, por desproporção } \\
\text { entre o sinal apresentado e o nível de do- } \\
\text { mínio da habilidade requerida para sua de- } \\
\text { tecção }\end{array}$ \\
\hline $\begin{array}{l}\text { Adequação para } \\
\text { ensino }\end{array}$ & $\begin{array}{l}\text { Em geral alta, pois apresentam pro- } \\
\text { blemas reais e concretos }\end{array}$ & $\begin{array}{l}\text { Pode ser baixa, por causar constrangimen- } \\
\text { to ao estudante e desconforto ao paciente }\end{array}$ \\
\hline Credibilidade & $\begin{array}{l}\text { Muito alta, pois representam proble- } \\
\text { mas reais }\end{array}$ & $\begin{array}{l}\text { Ocasionalmente baixa, por desconforto ou } \\
\text { má vontade do paciente }\end{array}$ \\
\hline $\begin{array}{l}\text { Adequação para } \\
\text { avaliação }\end{array}$ & $\begin{array}{l}\text { Alta para avaliação formativa de pe- } \\
\text { queno número de estudantes }\end{array}$ & $\begin{array}{l}\text { Baixa para avaliação somativa em que gran- } \\
\text { de número de estudantes devem ser ava- } \\
\text { liados nas mesmas condições }\end{array}$ \\
\hline Aceitabilidade & $\begin{array}{l}\text { Alta, por se tratar de recurso tradi- } \\
\text { cional }\end{array}$ & $\begin{array}{l}\text { Às vezes baixa, por questionamentos de } \\
\text { ordem ética }\end{array}$ \\
\hline Acesso & $\begin{array}{l}\text { Variável, dependente do acaso e das } \\
\text { condições clínicas do paciente }\end{array}$ & $\begin{array}{l}\text { Às vezes difícil, pois nem sempre o paci- } \\
\text { ente "desejado" está disponível }\end{array}$ \\
\hline $\begin{array}{l}\text { Custo operacional e } \\
\text { financeiro }\end{array}$ & $\begin{array}{l}\text { Baixo, pois se encontra embutido no } \\
\text { custo da assistência à saúde }\end{array}$ & $\begin{array}{l}\text { Pode ser alto, se o paciente tiver que ser } \\
\text { recrutado e convidado a participar das ati- } \\
\text { vidades }\end{array}$ \\
\hline
\end{tabular}

tornar as desvantagens da utilização dos pacientes reais $^{2 / 5}$. Destaque-se que a utilização de simulações, em geral, vem sendo considerada como um poderoso fator de melhora do desempenho profissional, sem a contrapartida de oferecer risco ou desconforto aos pacientes, em função, sobretudo, da possibilidade que oferece de se aprender com os erros, recebendo as correções apropriadas ${ }^{5,6}$.

\section{3- HISTÓRICO E DIFUSÃO}

A origem do desenvolvimento da técnica de utilização de pacientes simulados no ensino e na avaliação das habilidades clínicas associa-se fortemente ao nome do Professor Howard S. Barrows, atualmente, na Southern Illinois School of Medicine (Springfield, Illinois, Estados Unidos da América) e se encontra detalhadamente descrita em uma publicação específi$\mathrm{ca}^{3}$. Trabalhando em um hospital neurológico ligado à
Universidade Columbia, em New York, no início da década de 1960, Barrows logo soube que o seu superior, Dr. David Seegal, fazia questão de avaliar os seus residentes e realizava, como parte desta avaliação, a observação detalhada de como cada um deles tomava uma história clínica completa e realizava o exame neurológico sistematizado. Ao se engajar nestas atividades de avaliação, Barrows observou que: a) alguns pacientes podem se sentir muito desconfortáveis quando participam de atividades educacionais; b) que pacientes não preparados podem modificar sua história, e até mesmo simular sinais, involuntariamente, ou, mesmo, para favorecer ou prejudicar candidatos em exames de habilidades clínicas.

Mudando-se para um hospital geral de Los Angeles, ligado à Southern California School of Medicine, Barrows envolveu-se na produção de um filme didático, para ilustrar como é feito o exame neurológico. Esta experiência lhe mostrou que pessoas 
normais, modelos profissionais ou atores podiam ser treinados para desempenhar, com bastante realismo, o papel de pacientes, aprendendo a relatar sintomas e a simular sinais ou quadros neurológicos. Estas observações o estimularam a investir, de modo sistemático e intensivo no treinamento de pessoas normais para protagonizar "casos clínicos", para fins de ensino ou de avaliação ${ }^{7}$, para o que teve grande apoio do Professor Stephen Abrahamson, um renomado educador médico norte-americano. Esta atividade foi favorecida pela mudança de Barrows para Hamilton, Ontario, Canadá, onde compôs o grupo fundador da McMaster University, a primeira escola que adotou a aprendizagem baseada em problemas, como modelo curricular. Neste modelo inovador, pode ele aplicar intensivamente o recurso metodológico do paciente simulado para expor estudantes a condições clínicas relevantes, mas que não são encontradas com frequiência na prática clínica, bem como para o treinamento das habilidades clínicas, antes da sua aplicação em pacientes reais ${ }^{3}$. Foi nesta instituição que, anos mais tarde, o Professor Geoff Norman propôs a substituição do termo "paciente simulado" por "paciente padronizado", de modo a ressaltar a propriedade ímpar do método, de permitir a exposição dos estudantes às mesmas situações clínicas, nas mesmas condições (padronização), seja para fins de ensino, seja para fins de avaliação ${ }^{2,3}$.

Deve ser, também, registrado que, na mesma época (décadas de 1960 e 70), educadores médicos britânicos já utilizavam, sobretudo em exames, pacientes reais "preparados" ou "ensaiados", empregando conceitos e métodos semelhantes aos que formam, hoje, a base do treinamento de pacientes $\operatorname{simulados}^{2,8}$.

A difusão de modelos curriculares mais "centrados" nas necessidades dos estudantes ${ }^{9}$, bem como a disseminação de exames clínicos objetivos estrutu$\operatorname{rados}^{8}$, que permitem a avaliação de grande número de examinandos nas mesmas condições, passaram a se constituir em fatores favorecedores para a utilização intensiva, em todo o mundo, dos métodos envolvendo pacientes simulados. Em especial, a proposta de re-introdução, no final do século XX, de exame prático com paciente padronizado pelo National Board of Medical Examiners dos Estados Unidos da América, empregando modelo estruturado e padronizado para a fase 2 da etapa clínica ${ }^{10}$, constituiu um importante fator de difusão deste recurso, uma vez que esta medida foi precedida por amplo programa de pesquisa sobre suas características. O alcance desta difusão, especialmente nos Estados Unidos da América e no
Canadá, pode ser apreciado pela informação de que $57 \%$ das escolas médicas norte-americanas incluem em seu currículo atividades educacionais com pacientes simulados ${ }^{11}$. Este alcance pode, também, se aquilatado pela existência de uma associação acadêmica voltada aos profissionais envolvidos com o recrutamento e o treinamento de pacientes simulados ou padronizados, a American Association of Standardized Patient Educators (www.aspeducators.org).

No Brasil, a utilização deste recurso educacional pode ser reconhecida, em graus variáveis, em poucas escolas médicas, sobretudo as que adotam o modelo curricular do "aprendizado baseado em problemas", mas também em escolas mais tradicionais, como as duas instituições ligadas à Universidade de São Paulo (USP), localizadas em São Paulo e em Ribeirão Preto. No entanto, é difícil precisar quando e onde se iniciou a utilização de pacientes simulados no país, pois são escassos os relatos publicados sobre o tema. $\mathrm{Na}$ Faculdade de Medicina de Ribeirão Preto da Universidade de São Paulo (FMRP-USP), iniciamos a utilização de pacientes padronizados no início da década de 1990, primeiramente na avaliação final da disciplina de Semiologia ${ }^{12}$ e, logo após, em um programa mais abrangente de avaliação da eficácia do currículo com base nos dados de desempenho dos formandos, que incluía a realização de exames clínicos objetivos estruturados ${ }^{13,14}$. Desde então, este recurso metodológico de aperfeiçoamento educacional vem sendo utilizado continuamente em nossa instituição, em pequena escala, mas sem interrupção, bem como em outras instituições do Estado $^{15}$.

\section{4- NOMENCLATURA}

As simulações de situações clínicas para fins de ensino ou de avaliação educacional apresentam atualmente grande difusão e alta diversidade de recursos (Quadro 3), o que implica na utilização de diferentes designações para técnicas semelhantes. Assim, torna-se útil rever a nomenclatura que tem sido mais habitualmente aplicada para os vários tipos de simulação clínica envolvendo pessoas. Nesta breve revisão adotam-se os termos propostos pelo próprio Prof. Barrows ${ }^{3}$, bem como o de revisão crítica do tema, feito por autores britânicos ${ }^{2}$, que consideram as seguintes denominações:

- Paciente programado ou paciente ensaiado - paciente real treinado para protagonizar o seu próprio problema clínico, às vezes com pequenas modifica- 
ções propositais de algumas características, visando obter melhor adequação educacional; este recurso é usualmente empregado em avaliações de habilidades clínicas;

- Protagonista (role player) - estudante, professor ou instrutor que desempenha o papel de um dos integrantes da situação clínica: médico, paciente, familiar, acompanhante, membro da equipe profissional, etc., para fins de ensino e de treinamento;

- Pacientes simulados - designação genérica aplicada a pessoas normais que são treinadas para fazerem o papel de pacientes para fins de ensino ou de avaliação;

- Paciente padronizado - designação mais ampla, pois se aplica tanto a pessoas normais (pacientes simulados), como a pacientes reais, devidamente preparados para protagonizar "casos clínicos". No entanto, há a tendência de se chamar de pacientes padronizados, as pessoas que participam de avaliações, pois é neste contexto que a padronização, ou seja, o desempenhar o papel sempre da mesma maneira, é essencial para o bom cumprimento da função;

- Paciente ator - ator (amador ou profissional) que desempenha a função de paciente simulado ou padronizado em situações clínicas que exigem alto grau de dramaticidade;

- Instrutor prático - pessoa normal recrutada e treinada para voluntariamente submeter-se passivamente a exames retal ou ginecológico em situações de ensino específicas;

- Paciente instrutor - paciente real treinado para participar ativamente de situações de ensino, em geral de habilidades do exame físico, incluindo a apreciação crítica das tarefas clínicas realizadas pelos estudantes;

- Substituto de paciente - termo genérico empregado para todas as simulações instrumentais que não envolvem pessoas: modelos anatômicos, manequins, fitas de áudio, simulações computadorizadas;

- Paciente virtual - técnica computadorizada avançada de substituição de paciente, utilizada no aprendizado eletrônico de habilidades de comunicação, que permite a geração realística, a partir de filmes digitalizados, em ambiente de "realidade virtual", de "pacientes" com capacidade de interagir com o estudante ${ }^{16,17}$.

\section{5- VANTAGENS DO USO DE PACIENTES SIMULADOS}

Antes de qualquer consideração que pode ser feita sobre as vantagens do uso de pacientes simulados, sobretudo no ensino das habilidades clínicas, deve ser enfatizado que este recurso não substitui o paciente real, que sempre terá um papel central na educação dos profissionais da área da saúde. A utilização dos pacientes simulados, em suas várias modalidades (Quadro 3) deve ser vista como complementar ao uso dos pacientes reais, em especial, antecedendo a prática mais intensiva com eles, de modo a permitir que as atividades educacionais envolvendo o binômio estudante - paciente sejam desenvolvidas com mais segurança e menor desgaste para as partes envolvidas. Em outras palavras, a prática com pacientes simulados pode melhor preparar o estudante para o trabalho com pacientes reais.

Quadro 3. Principais recursos de simulação clínica envolvendo pessoas, empregados no ensino e na avaliação de competências (ver texto para nomenclatura)

RECURSOS

- Paciente programado ou ensaiado

- Protagonizador (role player)

- Paciente simulado (padronizado)

- Paciente instrutor

- Substituto de paciente

- Paciente virtual

O exame das vantagens da utilização de pacientes simulados deve, também, ser precedido de considerações sobre os principais problemas usualmente associados ao emprego das técnicas tradicionais no ensino das habilidades de comunicação para os futuros profissionais da saúde (Quadro 4). Entende-se, aqui, por técnica tradicional, a abordagem usualmente empregada em muitas escolas, que envolve uma sequiência de procedimentos, como: a) ensinar o estudante as bases conceituais da tarefa, por meio de aulas e leituras sobre seus aspectos teóricos; b) efetuar demonstrações sobre o que deve ser aprendido; esta etapa é usualmente realizada "ao vivo", com pacientes reais, podendo ser ou não precedida por exibições de filmes 
demonstrativos; c) estimular o estudante a praticar a tarefa, individualmente ou em duplas; esta etapa é necessariamente supervisionada, embora, freqüentemente, a atividade do estudante em seus exercícios práticos não é de fato observada pelo supervisor e depende do relato feito sobre a tarefa ${ }^{1}$; d) realizar avaliação somativa final para concluir se o estudante aprendeu ou não a tarefa; isto é usualmente feito por meio de provas práticas não estruturadas e, não raro, apenas por meio de provas teóricas.

O Quadro 4 relaciona os principais problemas associados a esta abordagem mais tradicional, que incluem, entre outros: a) o caráter mais passivo da experiência, uma vez que o aprendizado ativo é pouco explorado; b) a assimetria do envolvimento individual do estudante, que depende de vários fatores não controlados, como personalidade, interação com os colegas, interesse, disposição, etc.; c) o elenco relativamente limitado de situações a que o estudante é exposto, que, por sua vez, é altamente dependente da disponibilidade de pacientes; d) a heterogeneidade de oportunidades oferecidas aos estudantes, também dependente da disponibilidade de pacientes; e) a carência de comentários formativos, sobretudo nas situações em que as atividades práticas dos estudantes não são efetivamente observadas; f) o constrangimento imposto aos estudantes, que têm que interagir com os pacientes mesmo sabendo que ainda não detêm o nível mínimo de proficiência para a execução da tarefa; g) o desgaste que as atividades podem representar para os pacientes reais.

Quadro 4. Principais problemas associados ao emprego das técnicas tradicionais no ensino das habilidades de comunicação para os futuros profissionais da saúde

PROBLEMAS

- Aprendizado ativo pouco explorado

- Envolvimento individual assimétrico

- Elenco limitado de situações

- Heterogeneidade de oportunidades

- Inibição ou constrangimento para os participantes

- Supervisão à distância (sem observação)

- Carência de feedback

- Desgaste para pacientes reais
Em termos genéricos, não é impróprio afirmar que a utilização das simulações clínicas, desde os recursos mais simples até os mais elaborados (Quadro 3 ), pode contribuir para contornar todos estes inconvenientes (Quadro 4). Pode oferecer condições de aprendizado mais ativo, maior envolvimento individual dos estudantes, elenco mais amplo de situações clínicas a serem exploradas, maior homogeneidade nas oportunidades a que os alunos podem ser expostos, melhores condições de observação e oferecimento de comentários construtivos, menor constrangimento para os participantes e menor desgaste para os pacientes reais. Outras vantagens genéricas assinaladas em revisão sistemática do tema ${ }^{6}$ são a de permitir as devidas repetições da tarefa clínica, a de possibilitar a prática das tarefas no nível desejado de complexidade, dentro de espectro praticamente ilimitado de situações clínicas, bem como a de prover condições para intervenções voltadas a integrar temas de diferentes áreas do currículo.

Em termos mais específicos, a utilização das simulações clínicas em geral e a dos pacientes simulados ou padronizados, em particular, pode oferecer um conjunto de vantagens, que são apresentadas na Quadro 5.

\section{6- DESVANTAGENS DO USO DE PACIENTES SIMULADOS}

O recrutamento, a seleção e o treinamento de pacientes simulados constituem processos muito trabalhosos e demorados, mesmo com a utilização em pequena escala deste tipo de recurso. Quando se planeja a utilização em grande escala, como ocorre em escolas em que o currículo prevê a participação sistemática de pacientes simulados nas atividades educacionais ou em centros de avaliação de habilidades e competências clínicas, além do dispêndio de esforços e do tempo, há que se considerar o alto custo financeiro associado. Este custo elevado se distribui na remuneração dos pacientes simulados ou no ressarcimento das suas despesas, quando se trabalha com voluntários. Porem, o seu principal componente é o que se associa à necessidade de dispor de pessoas treinadas para administrar um programa específico e para cumprir as tarefas e funções necessárias ao recrutamento, seleção e treinamento dos pacientes simulados.

Sob o prisma educacional, o uso de pacientes simulados no ensino e em exames ainda enfrenta resistências conceituais de profissionais que julgam, equivocadamente, estarem eles substituindo os pacientes 
Quadro 5: Vantagens do emprego de pacientes simulados

\begin{tabular}{|c|c|}
\hline VANTAGENS & COMENTÁRIOS \\
\hline Disponibilidade & $\begin{array}{l}\text { Pessoas confiáveis recrutadas para atuar como pacientes simulados estão } \\
\text { sempre disponíveis; várias pessoas podem ser treinadas para desempenhar } \\
\text { o mesmo papel }\end{array}$ \\
\hline Consistência & $\begin{array}{l}\text { Pacientes simulados bem treinados desempenham com realismo, acurácia e } \\
\text { de forma reprodutível seus papéis }\end{array}$ \\
\hline Padronização & $\begin{array}{l}\text { O paciente simulado bem treinado desempenha o mesmo papel sempre da } \\
\text { mesma maneira }\end{array}$ \\
\hline Multiplicação & Várias pessoas podem ser treinadas para desempenhar o mesmo papel \\
\hline Controle do nível de complexidade & $\begin{array}{l}\text { A mesma situação clínica ou problema pode ser apresentada em diferentes } \\
\text { níveis de complexidade, de modo a se adequar ao estágio de aprendizado do } \\
\text { estudante }\end{array}$ \\
\hline Adequação & $\begin{array}{l}\text { Pacientes simulados podem ser empregados no ensino e no treinamento } \\
\text { para atuação em situações em que seria impróprio utilizar pacientes reais }\end{array}$ \\
\hline Repetição da tarefa & $\begin{array}{l}\text { A mesma tarefa pode ser repetida inúmeras vezes pelo mesmo estudante ou } \\
\text { por outros empregando o mesmo paciente simulado }\end{array}$ \\
\hline Tolerabilidade & $\begin{array}{l}\text { Pacientes simulados suportam bem a repetição de tarefas, a sua execução } \\
\text { por vários estudantes ou a participação em sessões prolongadas }\end{array}$ \\
\hline Ensino & $\begin{array}{l}\text { Pacientes simulados podem ser instruídos para colaborar no ensino, fazen- } \\
\text { do correções ou emitindo comentários construtivos sobre a atuação do } \\
\text { estudante }\end{array}$ \\
\hline Avaliação & $\begin{array}{l}\text { Pacientes simulados podem ser treinados para avaliar a adequação da tarefa } \\
\text { desempenhada, seja fazendo comentários construtivos, seja colaborando } \\
\text { para a tomada de decisões }\end{array}$ \\
\hline
\end{tabular}

reais ${ }^{2}$. Dependendo das circunstâncias da sua utilização, a situação clínica da utilização dos pacientes simulados pode, de fato, carregar-se de certa artificialidade, o que pode comprometer a acurácia e a credibilidade do desempenho do papel. Isto ocorre, naturalmente e com maior frequiência, em exames estruturados com participação manifesta de pacientes padronizados, o que exige que, no desempenho das tarefas clínicas solicitadas, o examinando exercite uma atitude conhecida como "suspensão do descrédito" ${ }^{18}$. As desvantagens ligadas à artificialidade e reduzidas acurácia e credibilidade no desempenho do papel acentuam-se, obviamente, quando o treinamento do paciente simulado apresenta deficiências.

Uma importante desvantagem do emprego dos pacientes simulados relaciona-se a sua utilização para ensino e avaliação de habilidades relacionadas ao exame físico. Ainda que pessoas normais possam ser preparadas ou treinadas para simular uma variedade de sinais físicos, sobretudo os que podem ser detectados ao exame neurológico (Quadro 6), o elenco de alterações é bastante limitado. Além disso, o risco de artificialidade aumenta, visto que é impossível simular a maioria das anormalidades a que os estudantes devem ser expostos.

Um resumo das principais desvantagens da utilização educacional de pacientes simulados é apresentado no Quadro 7.

\section{7- PARTICULARIDADES DA UTILIZAÇÃO DE PACIENTES SIMULADOS NO ENSINO E NA AVALIAÇÃO}

No que se refere ao emprego de pacientes simulados no ensino, é o treinamento das habilidades clínicas que envolvem a interação e a comunicação com o paciente, incluindo a obtenção da história clínica e o fornecimento de informações e orientações, o que constitui a situação ideal de utilização deste recurso. 
Quadro 6. Principais sinais físicos que podem ser simulados por pessoas normais adequadamente treinadas (modificado de Barrows, 1993 . ${ }^{3}$. Algumas simulações exigem o emprego de recursos maquiagem (m)

\begin{tabular}{ll}
\hline & SINAIS FíSICOS \\
\hline Gerais & Neurológicos \\
Ferimentos (m) & Afasia \\
Icterícia (m) & Ataxia \\
Obstrução de vias aéreas & Atetose \\
Pneumotórax & Coma \\
Respiração anormal & Confusão \\
(Kussmaul, Cheyne-Stokes) & Corea \\
Rigidez abdominal (abdome agudo) & Disartria \\
Rigidez articular & Hemiparesia \\
Sibilância & Hiper-reflexia \\
Sopro carotídeo & Incoordenação \\
Sopro tireóideo & Paralisia facial \\
Taquicardia & Rigidez de nuca \\
Taquipnéia & Sinal de Brudzinski \\
Tosse & Sinal de Babinski \\
Traumatismos (m) & Tremores \\
Vômitos & \\
\hline
\end{tabular}

Quadro 7: Principais desvantagens da utilização de pacientes simulados no ensino e na avaliação de habilidades clínicas

DESVANTAGENS

\section{Aceitabilidade}

Credibilidade

Adequação para exame físico

Custo financeiro

Custo operacional
COMENTÁRIOS

Alguns profissionais julgam, equivocadamente, que não é lícito utilizar pessoas normais simulando situações clínicas ou substituindo os pacientes reais

Em algumas circunstâncias, o desempenho do papel é pouco acurado ou exige a atitude de "suspensão do descrédito" (ver texto)

A maioria dos sinais físicos é impossível de ser simulada; a simulação de muitos sinais pode se associar a artificialidade, comprometendo a credibilidade

Sempre há a necessidade de remunerar o paciente simulado ou, ao menos, de ressarcir as despesas relacionadas a sua participação

Sempre elevado, pois envolve recrutamento, seleção e treinamento 
No aprendizado da tomada da história clínica, os pacientes simulados podem ser empregados de modo genérico ou, então, em exercícios de focalização, para que o estudante obtenha prática em tarefas específicas. Estas incluem o delineamento do quadro clínico e sua evolução, a caracterização detalhada dos sintomas, o interrogatório sistematizado de queixas pertinentes aos diversos órgãos e aparelhos e a caracterização dos antecedentes pessoais e familiares.

Os pacientes simulados podem ainda ser utilizados para o treinamento das habilidades de informar sobre a natureza do sintoma ou da doença subjacente, sobre os procedimentos diagnósticos ou terapêuticos que serão necessários, informar sobre as particularidades do uso de medicamentos e aplicar recomendações sobre como conseguir maior adesão do paciente.

No treinamento das habilidades de comunicação, além da possibilidade de simular um elenco muito diversificado de situações e de modificar o nível de complexidade de modo a atender ao desejado para o estágio de formação do estudante (Quadro 5), é possível delinear atividades individuais ou em pequenos grupos de modo a fomentar o relacionamento empático com o paciente ${ }^{19}$.

Um aspecto da utilização no ensino dos pacientes simulados que vale ressaltar é a possibilidade de planejar adequadamente as situações clínicas a que o estudante deve ser exposto e garantir que todos o sejam nas condições desejadas.

Em todas as atividades de ensino e treinamento de habilidades de comunicação envolvendo o uso de pacientes simulados, o engajamento ativo do estudante e os comentários formativos sobre a sua atuação têm grande importância no sentido de otimizar o aprendizado. Estes comentários podem ser feitos pelo próprio paciente simulado, desde que adequadamente treinado para isto.

Alguns aspectos da utilização dos pacientes simulados no ensino são particularmente valiosos e merecem destaque. Entre estes, está a possibilidade do estudante iniciante praticar sem constrangimentos e pelo tempo que for necessário para se sentir confiante em trabalhar com pacientes reais. No que se refere aos constrangimentos, está a possibilidade de abordar com o paciente simulado, tópicos "sensíveis", como os relacionados à atividade sexual e seus antecedentes.

Ainda que a utilização de pacientes simulados no aprendizado de habilidades de comunicação apresente as vantagens antes mencionadas, é no campo da avaliação que este recurso é muito mais utiliza- $\mathrm{do}^{2,3,10,12,15,18,20,21}$. A provável razão para isto é que o emprego de pacientes simulados (padronizados) oferecer condições ótimas de preenchimento dos requisitos de validade e fidedignidade que todo procedimento de avaliação deve ter ${ }^{20}$. Entende-se por validade o atributo de se avaliar exatamente o que se pretende, no contexto apropriado e no nível adequado de complexidade. Nos exames convencionais, o preenchimento do critério de validade é, com freqüência, ameaçado pela indisponibilidade de pacientes reais com as características desejadas. Já a fidedignidade diz respeito à consistência do julgamento que é efetuado, o que, por sua vez, depende da inclusão nos exames práticos de uma amostragem variada e representativa de casos e, sobretudo, da estabilidade do desempenho dos pacientes frente aos diversos examinandos. De novo, o emprego de pacientes padronizados oferece amplas possibilidades de preenchimento deste critério, em nítida vantagem sobre a utilização de pacientes reais.

É interessante salientar que vários estudos demonstraram que o desempenho dos pacientes padronizados bem treinados é realístico, acurado e consistente ${ }^{21}$.

\section{8- ASPECTOS PRÁTICOS DA UTILIZAÇÃO DE PACIENTES SIMULADOS: RECRUTA- MENTO E TREINAMENTO}

A utilização em larga escala em atividades de ensino, treinamento e avaliação previstas na estrutura curricular demanda a criação de um centro específicos de gerenciamento de um "banco" de pacientes simulados, que, em primeiro lugar deverá contar não só com orçamento apropriado, mas também com pessoal capacitado para as funções de recrutamento, seleção e, sobretudo, de treinamento dos candidatos ${ }^{22,23}$.

O emprego em menor escala, como o que vimos persistentemente fazendo, há mais de uma década, na FMRP-USP ${ }^{12}$, pode ser desenvolvido com menor dispêndio de recursos e de pessoal.

De qualquer modo, é recomendável prever o alcance da escala da utilização dos pacientes simulados, detalhando os procedimentos que serão seguidos para recrutamento, seleção, treinamento e efetivo emprego das pessoas em atividades educacionais de aprendizado e/ou de avaliação e submeter o plano à apreciação tanto do colegiado coordenador do curso ou programa como do comitê institucional de Ética local. 
Algumas características desejáveis nas pessoas que desempenharão as funções de paciente simulado $^{2}$ são apresentadas no Quadro 8. Deve-se procurar pessoas que tenham algum vínculo com a instituição, uma vez que isto favorece o entendimento da importância das atividades a serem desempenhadas. Entre estas pessoas, com vínculo direto, podem-se incluir funcionários administrativos, técnicos de laboratório, estudantes e profissionais da saúde de outras áreas. Entre as com vínculo indireto, incluem-se exalunos, ex-funcionários, familiares ou amigos de pessoas com vínculo direto com a instituição. Em qualquer um destes grupos, é desejável que a pessoa a ser recrutada não tenha contato próximo com os estudantes com quem entrarão em contato no desempenho da função de paciente simulado, o que é importante para minimizar eventual artificialidade. O recrutamento ou convite é usualmente feito, com a devida antecedência, por meio de contatos pessoais ou telefônicos, após se obter informações que a pessoa detém as características desejadas. Dentre estas, estão a disposição e a boa vontade de participar, com espírito construtivo e em caráter de voluntariado, e a disponibilidade para estar presente quando necessário para as sessões de treinamento e, especialmente, para atuar no ensino e na avaliação. Um requisito específico muito valioso é a pessoa ter, como característica pessoal, argúcia e vivacidade para, de um lado, entender a importância da sua participação e a natureza do papel que irá desempenhar e, por outro lado, desempenhar satisfatoriamente este papel em situações inesperadas, como, por exemplo, para responder a perguntas imprevisíveis.

O treinamento dos pacientes simulados pode ser feito com grau variável de elaboração, mas a adesão

Quadro 8: Características desejáveis em candidatos a atuar em simulações clínicas

\section{CARACTERÍSTICAS}

- Vínculo direto ou indireto com a instituição

- Ausência de contato próximo com os estudantes

- Disposição e boa vontade

- Disponibilidade

- Capacidade de desempenhar o papel

- Idade e sexo compatíveis com o papel

- Argúcia e vivacidade a um conjunto de procedimentos relativamente simples $^{2,24}$, embora fiéis a recomendações incontestáveis $^{25}$, produz bons resultados. Estes procedimentos estão sumarizados no Quadro 9. O treinamento iniciase com uma conversa descontraída onde se averigua o entendimento que a pessoa tem da sua participação como paciente simulado e se verificam as condições de disposição e de disponibilidade para o treinamento e para a atuação, propriamente dita. Em seguida explica-se, em detalhes, como o papel deve ser desempenhado e se adiantam pontos mais importantes do roteiro do "caso", previamente redigido. A seguir, é dado o tempo suficiente para que a pessoa leia o roteiro e expresse suas dúvidas sobre o papel. Neste momento, o roteiro é discutido e se verifica se contem semelhanças ou dessemelhanças importantes com a biografia da pessoa que fará o papel. Dependendo do juízo conjunto do treinador e do candidato, pode ser feita a inclusão, no roteiro, das modificações pertinentes, de modo a facilitar o desempenho do papel, sem prejuízo do essencial à sua adequação às finalidades pretendidas. Este primeiro conjunto de procedimentos é cumprido em uma primeira sessão que deve durar menos que uma hora.

Após esta primeira sessão, o paciente deve memorizar o conteúdo do roteiro, o que é avaliado no

Quadro 9: Seqüência simplificada de procedimentos para o treinamento de pacientes simulados para atuação no ensino e na avaliação de habilidades clínicas

PROCEDIMENTOS

- Explicação detalhada sobre o papel

- Leitura do roteiro escrito

- Discussão do roteiro e inclusão das modificações pertinentes

- Memorização do roteiro

- Avaliação do entendimento da situação e do domínio do roteiro

- Primeira simulação com o treinador

- Correções das impropriedades

- Segunda simulação com o treinador

- Ajustes adicionais

- Simulação com "estudante ou profissional simulado"

- Ajustes finais 
início da segunda sessão do treinamento, junto com a verificação final do entendimento da situação e do papel. Feito isto, o paciente simulado está em condições de se submeter à primeira simulação, feita com o treinador desempenhando o papel do estudante ou examinando. Após as correções das impropriedades detectadas na primeira simulação, uma segunda simulação é feita ainda com o treinador, o que pode ser repetido mais uma ou duas vezes, se necessário.

Antes da atuação propriamente dita, uma última simulação deve ser feita, agora com uma terceira pessoa fazendo o papel de "estudante ou profissional simulado", sob a observação do treinador, que orientará o paciente simulado nos ajustes finais ao papel.

Este conjunto de procedimentos pode ser desenvolvido em duas ou três sessões, que tomam, seguramente, menos de três horas e tem alta probabilidade de resultar em bom desempenho do paciente simulado em sua participação nas atividades de ensino ou de avaliação de habilidades clínicas.

\section{9- CONCLUSÃO}

A utilização de pessoas normais desempenhando o papel de pacientes constitui um meio valioso de contornar os inconvenientes da utilização dos pacientes reais no ensino e na avaliação de habilidades clínicas e de preparar adequadamente os estudantes para o trabalho mais seguro e menos desgastante com os pacientes. O emprego desta técnica favorece o aprendizado mais ativo, permite repetições das tarefas clínicas e facilita a correção imediata dos defeitos na sua execução. Além disso, a utilização de pacientes simulados permite expor os estudantes a ampla variedade de situações clínicas, bem como possibilita conduzir avaliações tecnicamente mais adequadas. Longe de constituir recurso complicado e dispendioso, o recrutamento, a seleção e o treinamento de pessoas normais para atuar como pacientes podem ser feitos com custo reduzido e seguindo procedimentos relativamente simples.

Troncon LEA. Utilization of simulated patients for clinical skills teaching and assessment. Medicina (Ribeirão Preto) 2007; 40 (2): 180-91.

Abstract: The utilization of normal people portraying patients for clinical skills teaching and assessment has growing worldwide for the last decades, due to its capability to overcome the inconveniences associated to the use of real patients. As far as teaching is concerned, interaction with simulated patients may precede work with real patients, thus allowing that clinical skills learning may occur in a safer and less uncomfortable environment for both students and patients. The utilization of simulated patients also favors active learning and uniformity of opportunities between learners, allow tasks to be repeated several times, with prompt correction of distortions. The utilization of simulated, standardized patients in examinations allows the inclusion of the relevant clinical problems and situations, at the appropriate complexity level, while permitting the assessment of large numbers of examinees in the same conditions, which favor the fulfillment of both validity and reliability criteria. This paper presents a brief discussion of the educational concepts underlying clinical skills teaching and assessment and the utilization of simulated patients. Comparisons of the main advantages and disadvantages of using real or simulated patients are presented. Practical aspects and hints on recruiting, selecting, training and using simulated, standardized patients for clinical skills learning and assessment are presented, based on the small scale, albeit persistent, experience gathered at Ribeirão Preto Faculty of Medicine, University of São Paulo, Brazil, in the last decade.

Keywords: Simulation. Patient Simulation. Standardized Patients. Clinical Competence. Education, Medical. 


\section{REFERÊNCIAS}

1 - Gordon J. ABC of learning and teaching in medicine: One to one teaching and feedback. BMJ 2003; 326: 543-5.

2 - Collins JP, Harden RM. Real patients, simulated patients and simulators in clinical examinations. Med Teach1998; 20: 50821.

3 - Barrows HS. An overview of the uses of standardized patients for teaching and evaluating clinical skills. Acad Med 1993; 68: 443-51.

4 - Ziv A, Wolpe PR, Small SD, Glick S. Simulation-based medical education: an ethical imperative. Acad Med 2003; 78:783-8.

5 - Ziv A, Ben-David S, Ziv M. Simulation based medical education: an opportunity to learn from errors. Med Teach 2005; 27:193-9.

6 - Issenberg SB, McGaghie WC, Petrusa ER, Lee Gordon D, Scalese RJ. Features and uses of high-fidelity medical simulations that lead to effective learning: a BEME systematic review. Med Teach 2005; 27: 10-28.

7 - Barrows HS, Abrahamson S. The programmed patient: a technique for appraising student performance in clinical neurology. J Med Educ 1964; 39: 802-5.

8 - Harden RM, Stevenson M, Downie WW, Wilson GM. Assessment of clinical competence using objective structured examination. Br Med J 1975: 1: 447-51.

9 - Harden RM, Sowden S, Dunn WR. Educational strategies in curriculum development: the SPICES model. Med Educ 1984; 18: 284-97.

10 - Papadakis MA. The Step 2 clinical skills examination. N Engl J Med 2004; 350: 1703-5.

11 - Kirch DG. A word from the President. AAMC Reporter February 2007, disponível em http://www.aamc.org/ newsroom/reporter/feb07/word.htm (acessado em 10 de junho de 2207)

12 - Troncon LEA, Foss NT, Voltarelli JC, Dantas RO. Avaliação de habilidades clínicas por exame objetivo estruturado por estações, com emprego de pacientes padronizados: uma aplicação no Brasil (Parte II). Rev Bras Educ Med 1996; 20: 53-60.

13 - Troncon LE, Figueiredo JF, Rodrigues MLV, Peres LC, Cianflone AR, Picinato CE, Colares MFA. Implantação de um programa de avaliação terminal do desempenho dos graduandos para estimar a eficácia do currículo na Faculdade de Medicina de Ribeirão Preto. Rev Assoc Med Bras 1999; 45: 217-24.
14 - Figueiredo JF, Troncon LE, Rodrigues MLV, Cianflone AR, Colares MFA, Peres LC, Piccinato CE. Effect of curriculum reform on graduating student performance. Med Teach 2004; 26: 244-9.

15 - Ward LS, Wanderley JS, Monte-Alegre S. Implantação de um Sistema de Avaliação Estruturada de Performance em Escola Médica: Resultados do Projeto-Piloto. Rev Bras Educ Med 2005; 29: 174-8.

16 - Stevens A, Hernandez J, Johnsen K, Dickerson R, Ray A, Harrison $\mathrm{C}$ et al. The use of virtual patients to teach medical students history taking and communication skills. Am J Surg 2006; 191: 806-11.

17 - Zary N, Johnson G, Boberg J, Fors UG. Development, implementation and pilot evaluation of a Web-based Virtual Patient Case Simulation environment-Web-SP. BMC Med Educ 2006; 6:10.

18 - Epstein RM. Assessment in Medical Education. N Engl J Med 2007; 356: 387-96.

19 - Boyle D, Dwinnell B, Platt F. Invite, listen, and summarize: a patient-centered communication technique. Acad Med 2005 80: 29-32.

20 - Wass V, Van der Vleuten C, Shatzer J, Jones R. Assessment of clinical competence. Lancet 2001, 357: 945-9.

21 - Colliver JA, Williams RG. Technical Issues: Test Application. Acad Med1993; 68: 454-60.

22 - Ker JS, Dowie A, Dowell J, Dewar G, Dent JA, Ramsay J, Benvie S, Bracher L, Jackson C. Twelve tips for developing and maintaining a simulated patient bank. Med Teach 2005; 27: 4-9.

23 - Adamo G. Simulated and standardized patients in OSCEs: achievements and challenges 1992-2003. Med Teach 2003; 25: 262-70.

24 - Troncon LEA, Foss NT, Voltarelli JC, Dantas RO. Avaliação de habilidades clínicas por exame objetivo estruturado por estações, com emprego de pacientes padronizados: Descrição de dois métodos (Parte I). Rev Bras Educ Med 1996; 20: $45-52$.

25 - Barrows HS. Simulated (standardized) patients and other human simulations. Chapel Hill North Carolina: Health Sciences Consortium; 1987. 